\title{
Development of Picosecond Laser for Dermatology Application: Its Changeable Pulse Width and Bundle Pulse Implementation
}

\author{
Won J. Yi, PhD \\ CTO, R\&D division, Jeisys Medical, \\ Gwangmyeong, Korea
}

Received March 18, 2020

Revised April 2, 2020

Accepted April 10, 2020

\section{Correspondence}

Won J. Yi

CTO, R\&D division, Jeisys Medical, Gwangmyeong 14241, Korea E-mail:wnsk597@gmail.com https://orcid.org/0000-0002-9202-5891

(C) Korean Society for Laser, Dermatology and Trichology

(c) This is an open access article distributed under the terms of the Creative Commons Attribution NonCommercial License (http://creativecommons.org/ licenses/by-nc/4.0) which permits unrestricted noncommercial use, distribution, and reproduction in any medium, provided the original work is properly cited.
Jei-Pico laser, originally developed by Jeisys Medical in Korea, uses a new electro-optics technology beyond the existing SBS and Microchip laser. The characteristic feature of Jei-Pico is the variable pulse width from 180 ps to 2000 ps. Another function of Jei-Pico laser is that it's capable of 15 bundle pulses in one pumping cycle. Jei-Pico is capable of pulse combination that can change the pulse width of each of the 15 bundle pulses. The seed laser has been made with electro-optics technology that has a gain factor of $\sim 9.0 \times$ $10^{9}$ through three optical amplifiers. The development of Jei-Pico is focused on Nd:YAG rod with 1064 and $532 \mathrm{~nm}$. Maybe this technology can be applied to $532 \mathrm{~nm}$ as a pumping source to the Ti:Sapphire or alexandrite rod to produce the $700 \mathrm{~nm} \sim$ wavelength. Jei-Pico has about $600 \mathrm{MW}$ for 1064 $\mathrm{nm}$ and $220 \mathrm{MW}$ for $532 \mathrm{~nm}$.

\section{Key words}

Electro-optics, Pulse-width, Bundle pulse, Gain factor, Optical amplifier, Picosecond laser, nanosecond laser

Supplementary video fille: This article contains supplementary material (It is available at https://youtu.be/z8Ylh2oZhIQ). 


\section{INTRODUCTION}

Jeisys has been developed the dermatology applications of picosecond laser, called "Jei-Pico". It is a new type of pico-laser that has a changeable pulse width, bundle pulse implementation and combination pulse function. The dermatology applications for picosecond laser have made huge improvement after Cynosure's PicoSure published in December, 2012 [1]. The wavelength of those picosecond lasers has $1064 \mathrm{~nm}, 532 \mathrm{~nm}$, and $755 \mathrm{~nm}$, and recently published $730 \mathrm{~nm}$ using Ti:Sapphire rod [2-5]. Most of the picosecond lasers have the typical laser pulse width. Each company laser has a little different pulse width, but they have their unique pulse width [6]. Most of the pico-lasers have multiple wavelengths, however, it doesn't have either changing the pulse duration or bundle function (multipulse) or pulse combination function. Most pico-lasers used in dermatology had been produced optical pumping technology by seed laser. There are two general methods for making picosecond seed lasers. One was the Stimulated Brillouin Scattering (SBS) pulse compression technology [712 ] and the other one was the microchip laser technology [10,13-18]. Microchip laser by the Diode Pumped Solid State (DPSS) laser was a relatively small resonator, which could be producing picosecond seed laser. Each company has its own microchip lasers technology than can produce unique picosecond pulse width. The microchip laser produced short-pulsed duration seed laser. The pulse duration of the microchip seed laser had depended on a resonator size, pumping source and characteristic of passive q-switch. Thereafter the pulse width of the microchip laser having a predetermined structure was fixed once.

The motivation of the development of the Jei-Pico laser: 1) breakthrough products to lead the domestic and global market, 2) development of products that can appeal to global markets beyond current technology, 3) representative's strong commitment to the development of new concepts that can lead the pico-laser market. Jeisys optics lab developed new concepts of medical pico-lasers for leading the global market. Jei-Pico is a picosecond laser with variable pulse width either bundle pulse function or pulse combination function. Original technology developed in Jeisys Korea. The laser head designed and fabricated in Jeisys Korea. Firmware and software developed in Jeisys Korea.

The seed laser production was used electro-optical conversion technology that surpasses the existing technology of SBS or microchip technology. The Jei-Pico laser head composed of a unit that generates a seed laser and a unit that optically amplified.

A diode driver is designed for driving seed laser diodes for fiber laser, time-resolved Spectroscopy, LIDAR applications. The drivers can power most type one butterfly packages. A variety of pulse width, power and control options are available. Jei-Pico is a picosecond laser with variable duration and has either bundle pulse function or pulse combination implementation. A seed laser user-adjustable pulse width capability as low as 180 ps up to 2,000 ps at output currents up to $1.1 \mathrm{~A}$, as shown in Fig. 1. The pulse width was measured 180 ps for 0.4 A, 500 ps for $0.55 \mathrm{~A}$, and 2000 ps for $1 \mathrm{~A}$ of adjusting built-in resistor.

It can change the pulse duration from 180 picosecond to 2,000 picoseconds. It can make bundle pulse up to 15 pulses and also have a function for pulse combination. A seed laser user-adjustable pulse width capability as low as $180 \mathrm{ps}$ up to $2,000 \mathrm{ps}$ at output currents up to $1.1 \mathrm{~A}$.

The oscillogram shows the bundle pulse modules as shown Fig. 2. The left image shows the five bundle pulses of the $180 \mathrm{ps}$ and the fives of the 2,000 ps. The center shows the five pulses of $450 \mathrm{ps}$ and fives of $2,000 \mathrm{ps}$. The right side shows the five pulses of $750 \mathrm{ps}$ and another five 2,000 ps. When using the bundle pulses, each pulse has a lower peak power. That's why we can control the unwanted LIOB (Laser Induced Optical Breakdown) effect, as it depends on the peak power, not pulse energy.
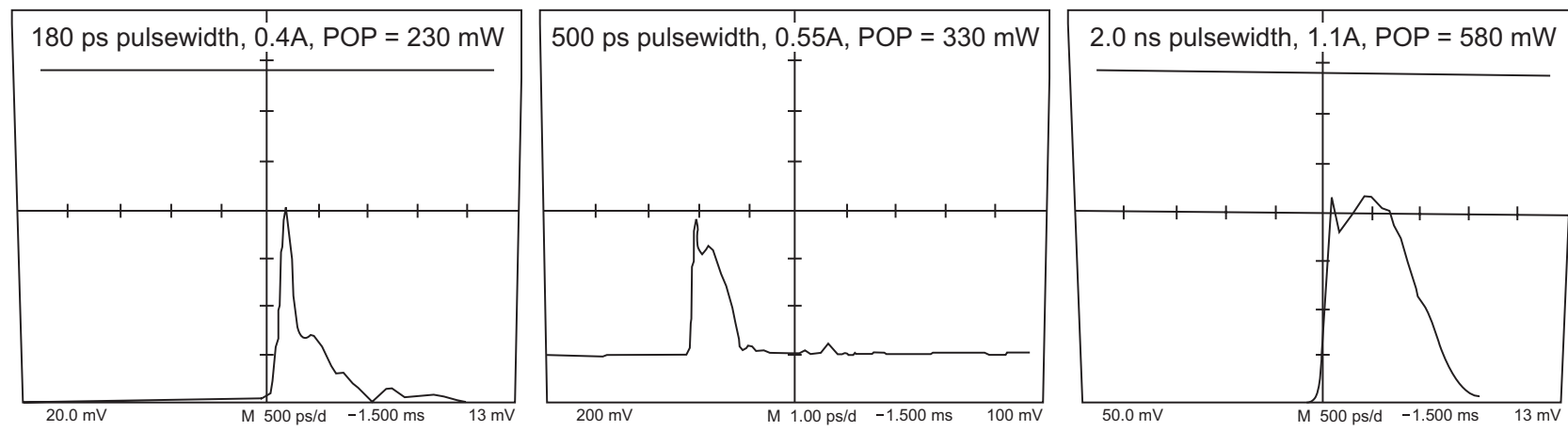

Fig. 1. Shows the variable pulse width with electro-optics conversion. 


\section{AESTHETICS}

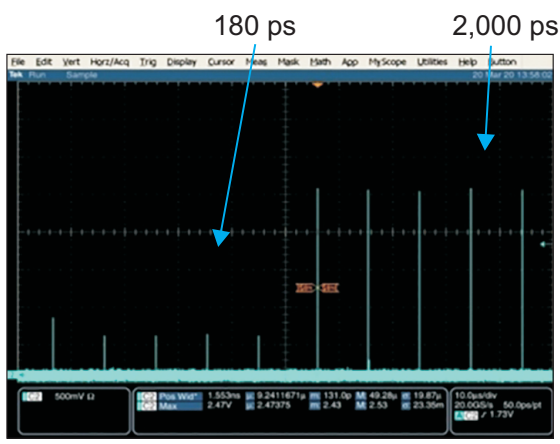

$180 \mathrm{ps}+2 \mathrm{~ns}$

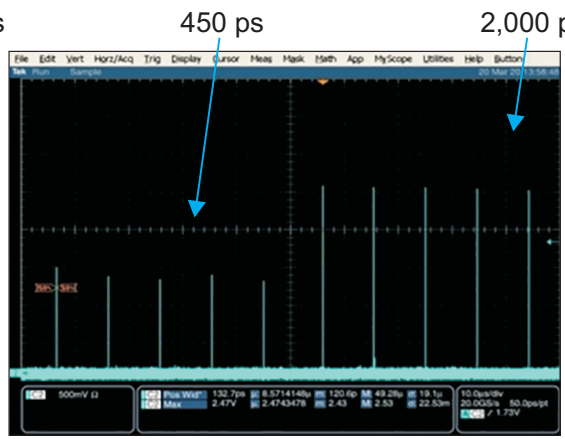

$450 \mathrm{ps}+2 \mathrm{~ns}$
750 ps

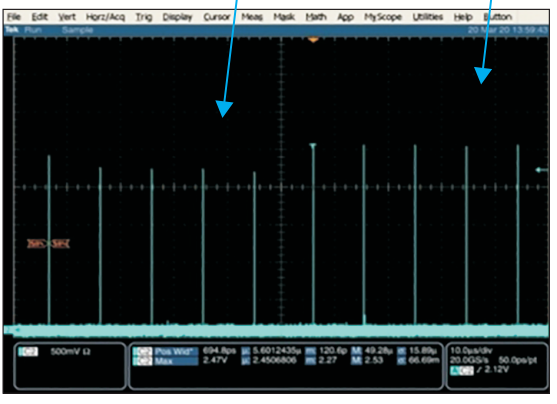

$750 \mathrm{ps}+2 \mathrm{~ns}$

Fig. 2. Shows the oscillogram for bundle pulses function with different pulse width variable pulse width with electro-optics conversion.

The unit for optical amplifiers consists of one preamplifier and two main amplifiers. Pre-amplified was for the optical amplifier with pumping by diode laser. The two main optical pumps are optical amplifiers pumped by Xe flash lamps. One flash-lamp pumping period has about 230 micro-second, this 230 micro-second called one pumping period. The seed laser of using SBS or microchip laser technology can be one optical pumping occurred with one seed laser in one pumping period. However, the seed laser of using electro-optics technology can be optical pumping up to 15 in one pumping cycle. It cannot make bundle or multipulse mode by the SBS and microchip technology. It is different from the repeat cycle. For example, ten hertz means ten cycles of 230 micro-second pumping cycle each second. The technology of generating the seed laser by electrooptics was totally different for making seed laser. It could make a variable pulse width, implementation of the bundle function and pulse combination function. We tried to make different methods for making seed laser and we developed totally different concept of the picosecond laser.

It can overcome clinical limitations with variable clinical applications. Also, short pulse width with high peak energy can produce Laser-Induced Optical Breakdown, LIOB. It can be induced unintentionally due to the high peak power. In our laser case, it can control output laser energy and can control each pulse's energy. Therefore we can control LIOB. That means, even the overall energy with very high peak pulse such as $800 \mathrm{~mJ}$ or $1 \mathrm{~J}$, peak power can be small even if overall energy is very high, for example, $1,000 \mathrm{~mJ}$ for 10-pulse can be divided into $100 \mathrm{~mJ}$ of each pulse. That is, we can be applied to variable clinic applications.

\section{CONCLUSION}

Optical pumping was a process that the light (Xe flash lamp) was used pumping energy to pump electron from the ground state to an excited state. It was used in laser to pump the active laser medium to achieve population inversion. Stimulated emission was the process by which a seed laser of a specific wavelength can interact with an excited electron fall to ground state energy level. Stimulated emission was the key: the process of generating two "cloned" photons: same frequency, phase, direction and polarization. It requires that there are more atoms in level E2 than in E1: N2 > N1 (Population Inversion). The optical amplifier did not have the oscillator. Optimum conditions must be established to achieve maximum pumping gain. Since there is no laser oscillator, as the seed laser passes through to the optical amplifier, more upper electrons falling to the ground state and established the best condition with the highest gain value. The laser has a typical characteristic. When you applied a certain wavelength to the optical amplifier with exactly the same pulse, it comes out except amplitude of the laser, which is a laser beam, but it doesn't have the resonator, only having an optical amplifier. To make a seed laser is important to develop the picosecond laser. When the low energy seed laser passes through the optical amplifier, a high amplified laser beam came out. The output laser had exactly the same wavelength and the same pulse width as the seed laser. That is, the specification of the seed laser and output laser beam were the specification except laser energy. For this reason, it is important to make a seed laser.

In order to have high output energy, it is necessary to have an optimal pumping gain value. Optimum conditions must be established to achieve maximum pumping gain. Since there is no laser oscillator, more of the upper state electrons must fall to the ground state as the seed laser passes through the pumped laser media. For the successful development of Jei-Pico laser, an optimal optical amplifier was designed to achieve the best optical gain.

The transition-cross section $\sigma(v)$ determines light-atom interaction. It is a function that characterizes the relative magnitude of the interaction of the atom with a photon of frequency $v[19,20]$. 


$$
\sigma(v)=\frac{\lambda 2}{8 \pi \mathrm{tspont}} \mathrm{g}(v)
$$

Probability density Wi: Given an atom with transition cross $=$ section $\sigma(\mathrm{v})[\mathrm{m} 2]$ and a photon-flux density $\phi\left[\mathrm{m}^{-2} \mathrm{~s}^{-1}\right]$ What is the probability Wi of a single photon being absorbed $[19,20]$ :

$$
\begin{aligned}
& \qquad \mathrm{Wi}=\phi \sigma(v)\left[=\mathrm{B}_{12} \rho(v)\right] \\
& \text { photons gained }=\left(\mathrm{N}_{2}-\mathrm{N}_{1}\right) \mathrm{W}_{\mathrm{i}}=N \mathrm{NW}_{\mathrm{i}}\left(\mathrm{m}^{-3} \mathrm{~s}^{-1}\right)
\end{aligned}
$$

Gain, bandwidth, phase shift, power source, nonlinearity and gain saturation, noise [19]

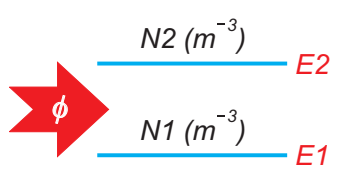

Where is the photon flux density $\left(\mathrm{m}^{-2} \mathrm{~s}^{-1}\right): \varphi=1 / \mathrm{hv}$, the gain coefficient $\left(\mathrm{m}^{-1}\right): \gamma(\mathrm{v})$, phase shift $\left(\mathrm{m}^{-1}\right): \phi(\mathrm{v})$ (Fig. 3) [21].

The photon flux density $\phi$ (photons/cm2-s) entering an incremental cylinder containing excited atoms grows to $\phi+$ $d \phi$ after length $d z, \phi=$ net atomic population $\times$ probability $x$ length, $\mathrm{Wi}=\phi \sigma\left(v, d \phi=N w_{i} d z=N \sigma(v) \phi(z) d z \gamma(v)=N \sigma(v)=\right.$ $N \frac{\lambda 2}{8 \pi t s p} g(v) ; \phi(z) d z=\phi(0) \exp \{\gamma(v) z\}, G(v)=\exp \{v(v) d\}:$ gain coefficient [19-21].

Block diagram of the Jei-Pico's laser head is shown in Fig. $4[22,23]$. The laser head consists of 4 units. The seed laser unit Electro-optical conversion. The seed laser includes electro-optical conversion. The electro-optical conversion technology is capable of variable laser pulse width, and it is possible to implement a bundled pulse function and pulse combination. The pulse width can be changed from 180 ps to $2,000 \mathrm{ps}$. The bundle function can be implemented up to 15 pulses. And the pulse combination function means that each pulse width can be implemented differently in the bundled pulse function. However, although there are such useful functions, the seed laser has a weakness with relatively small energy of tens of pico J. In order to overcome this weakness, a microchip optical amplifier1 was used. Optical amplifier 1 was used. As a pumping source, a diode laser was used, and the pumping laser beam transmitted through the optical fiber. In the development of Jei-Pico, a relatively small size microchip amplifier was used in consideration of laser head, space, and optical alignments.

Two amplifiers chamber were used optical pumping by xe flash lamp. Optical amplifiers using Xe lamps were most commonly used, such as Q-lasers. Each chamber has a Xe lamp with two Nd-Yag rods. The laser head uses one seed laser and three amplifiers. The laser head needs accurate

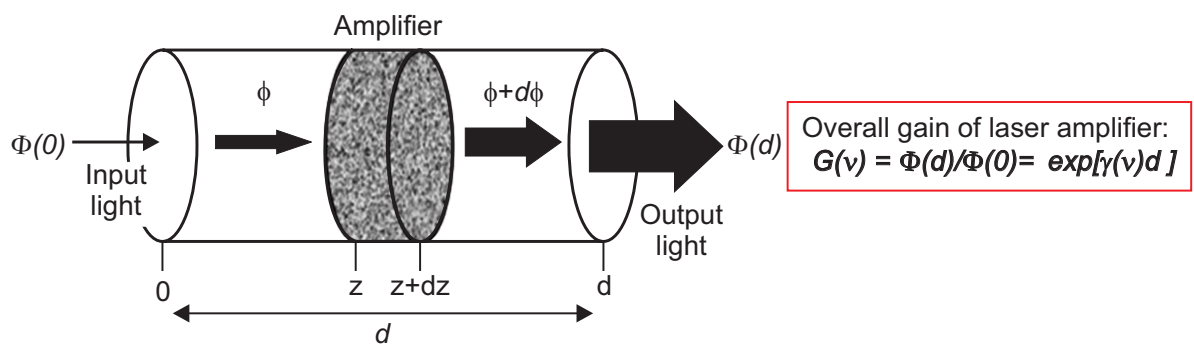

Fig. 3. The photon-flux density $\varphi$ (photons/cm2-s) increased gain, which excited electron optical gain to $\varphi+d \varphi$ pass through the length of $d z$. The overall gain of laser amplifier: $G(v)=\varphi(d) /$ $\varphi(0)=\exp [\gamma(v) d][21]$.

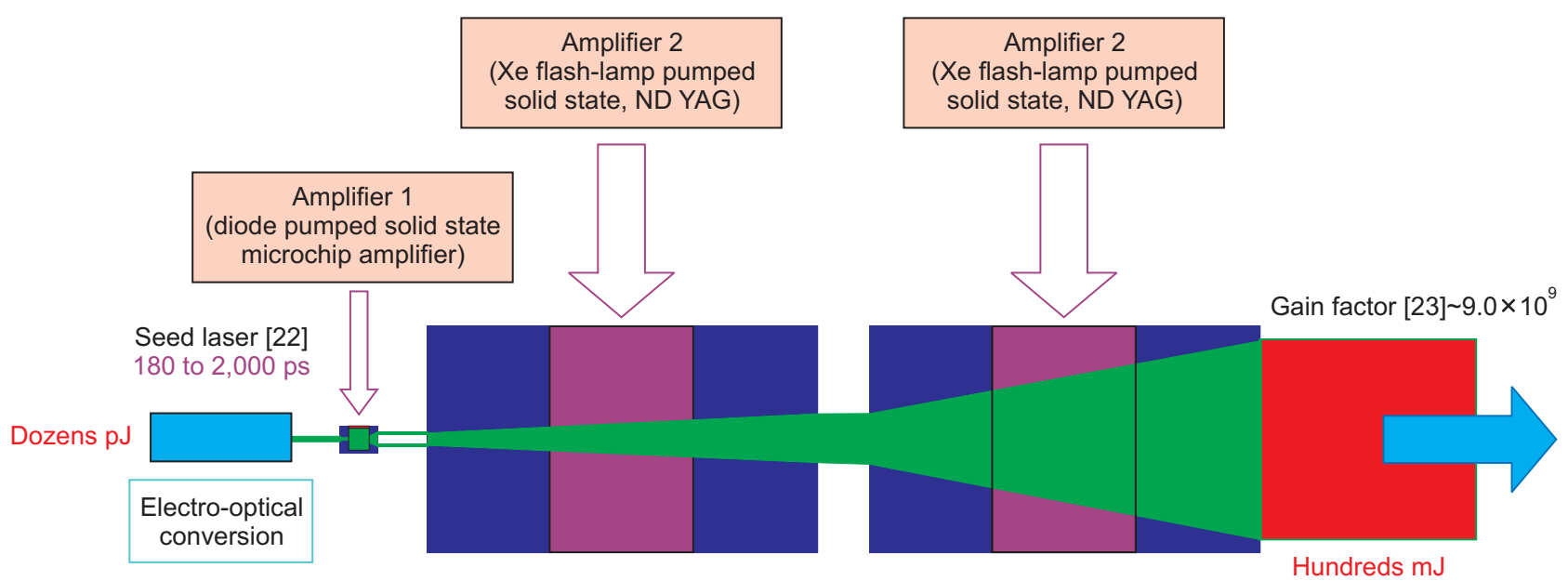

Fig. 4. Block diagram of the Jei-Pico's laser head. Laser head compose of seed laser with electro-optical conversion, amplifier 1 and two of amplifier 2. 
control of the seed laser oscillation time, the time for optical amplification of three different pumping sources, and the seed laser input time. The control board was also developed in the Jeisys optical laboratory (Fig. 5).

Table 1 shows the experimental results of the Jei-Pico laser. As of today (May, 2020) we have about $600 \mathrm{MW}$ for $1064 \mathrm{~nm}$ and $220 \mathrm{MW}$ for $503 \mathrm{~nm}$. It was not optimized yet. Our goal of the Jei-Pico is about $1 \mathrm{GW}$.

\section{DISCUSSION}

Jei-Pico started the "think different". As lasers have started to be applied in the medical field, the medical lasers have been applied to the medical field in a wide variety of output power. The diversity of laser power requires various types of laser which depend on the clinical application such as laser wavelength, pulse width, spatial power and out power level and output energy. It is necessary to develop medical devices along with the development of medical technology. Pico pulse lasers for the treatment of skin diseases have a fixed pulse width, which is limited to various clinical applications. Laser power, pulse width, pulse interval, pulse energy, and pulse peak power are very important for variables of skin treatment. The development of next-generation lasers with various functions such as laser output, changeable pulse widths, bundled pulses, and pulse combinations is required.

We started the "think different". It was to develop a laser that exceeds the limits of existing medical lasers, especially pico laser technology. It was the development of a laser that goes beyond the clinical limitations currently pico lasers for skin treatments. Jei-Pico laser has been developing it will be coming end of 2020. The feature of the Jei-Pico is shown in Table 2.

Jei-Pico adds new features that are not found in existing picosecond lasers. An important function is that the pulse width can be changed from 180 ps to 2000 ps. Since such a changing pulse width is possible for various clinical applications. Moreover, it is expected that new features can be ap-

Table 2. Jei-Pico features

\begin{tabular}{|c|c|c|}
\hline Subject & Value & Remark \\
\hline Variable pulse duration (ps) & $180-2,000$ & \\
\hline Pulse combined function & Up to 15 pulses & $\begin{array}{l}\text { Mixing the variable } \\
\text { pulse width }\end{array}$ \\
\hline Bundle pulsed function & Up to 15 pulses & $\begin{array}{l}\text { For one pumping } \\
\text { period }\end{array}$ \\
\hline Wavelength $(\mathrm{nm})$ & $\begin{array}{l}1064,532,755 \\
\text { (optional) }\end{array}$ & \\
\hline Repetition rate & 1 to $10 \mathrm{~Hz}$ & \\
\hline Beam divergence: & $\sim$ mRad & \\
\hline
\end{tabular}

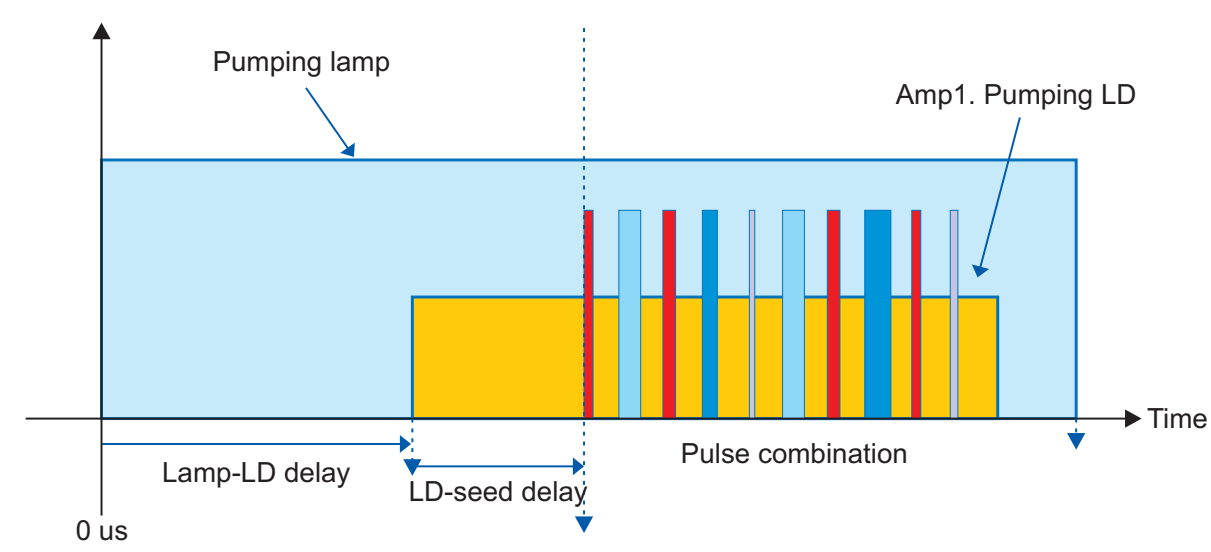

Fig. 5. Delay time of the lamp, amplifier 1 and laser diode. Delay time for the Bundle or combination pulses.

Table 1. Shows the experimental results of the Jei-Pico laser

\begin{tabular}{|c|c|c|c|c|c|c|c|c|c|c|c|}
\hline \multirow[b]{3}{*}{ Number of pulses } & & \multicolumn{10}{|c|}{ Measured value } \\
\hline & & \multicolumn{5}{|c|}{$532 \mathrm{~nm}$} & \multicolumn{5}{|c|}{$1064 \mathrm{~nm}$} \\
\hline & & 1 & 2 & 3 & 5 & 10 & 1 & 2 & 3 & 5 & 10 \\
\hline \multirow[t]{4}{*}{ Pulse energy (mJ) } & $180 \mathrm{ps}$ & 50 & 80 & 100 & 120 & 200 & 120 & 180 & 220 & 280 & 500 \\
\hline & $450 \mathrm{ps}$ & 100 & 160 & 200 & 230 & 300 & 250 & 350 & 400 & 500 & 700 \\
\hline & $750 \mathrm{ps}$ & 200 & 320 & 380 & & & 500 & 700 & 900 & & \\
\hline & $2,000 \mathrm{ps}$ & 300 & 500 & 650 & & & 700 & 1000 & 1200 & & \\
\hline
\end{tabular}


plied to new clinical applications. In the field of skin medical laser, when the laser is irradiated to the skin tissue, the reaction is classified into a photothermal and a photomechanical effect. In the treatment of skin tissue, a picosecond laser is used to produce a photomechanical effect that reduces heat damage to surrounding skin tissue and crushes and removes target particles. For the photomechanical effect, a nanosecond or picosecond laser is required. However, it is possible to create the best photomechanical effect by irradiating lasers of various pulse widths at the same energy according to the size of target particles and granules of small and large particles. A pico laser with a variable pulse width can be applied wide scope of the clinic with the photomechanical effect.

Up to 15 bundle pulse functions are possible. This bundle function is to minimize Laser Induced Optical Breakdown (LIOB) generated in picoseconds. In fact, when the pulse width was 200 ps in the laboratory, LIOB occurs in the air at about $30 \mathrm{~mJ}$ (pulse power: $150 \mathrm{MW}$ ) as shown in Fig. 6. As explained in the figure, $L I O B$ is generated in the air even at relatively low output energy (30 $\mathrm{mJ})$. When this laser is applied skin tissue, it is easily expecting formed the LIOB on the skin. It is not good for clinically performed when the unwanted LIOB occurs on the skin. The bundled pulse function can reduce each peak power even when irradiated with high pulse energy to the skin. Therefore, LIOB generation can be controlled. Since the interval of the bundle pulse can be adjusted from a few microseconds to tens of microseconds, therefore the photothermal effect can be minimized and the maximum photomechanical effect without LIOB.

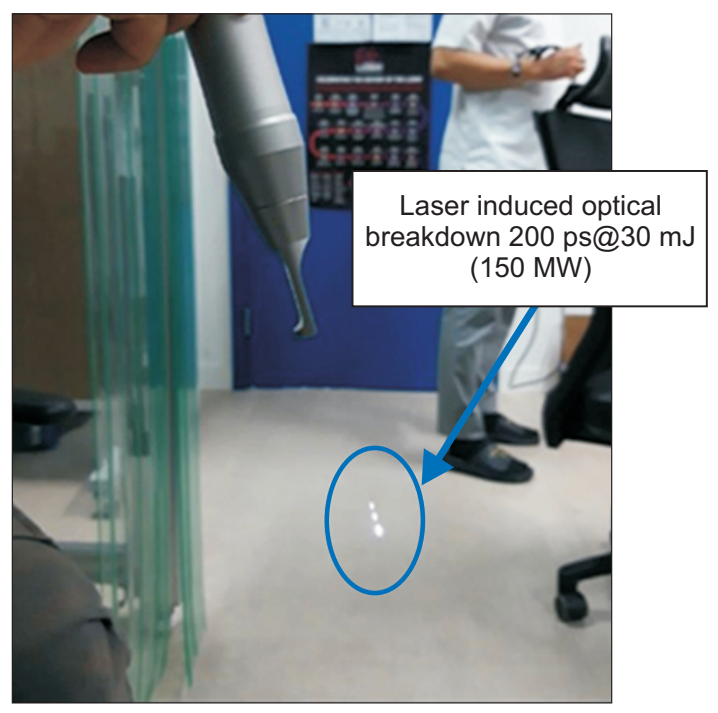

Fig. 6. Shows the LIOB in the air. Irradiation was 200 ps @ $300 \mathrm{~mJ}$ (150 MW pulse power).
The pulse combination function is also expected to be useful. Up to 15 pulses, the lasers of different pulse widths are used. It is expected that wider clinical applications than that of the bundled pulse function. In the pulse combination function, for example, when 2,000 ps and 200 ps pulses are used, it is expected that the clinical efficacy will occur compared to the existing pico-nano function.

So far, the development is focused on Nd:YAG rod with 1064 and $532 \mathrm{~nm}$. Maybe this technology can be applied to $532 \mathrm{~nm}$ as a pumping source to the Ti:Sapphire or alexandrite rod to produce the $700 \mathrm{~nm} \sim$ wavelength. The other way, my electro-optics seed laser can produce the seven hundreds- wavelength lasers, such as 750, 755, $760 \mathrm{~nm}$ using the alexandrite rod, or the Ti:Sapphire rod. Especially, the Ti:Sapphire rod has a big emission band. It can choose any seven-hundreds wavelength like 730, $755 \mathrm{~nm}$. Laser with eighty-hundreds wavelength is also possible.

\section{CONFLICT OF INTEREST}

No potential conflict of interest relevant to this article was reported.

\section{REFERENCES}

1. Cynosure, Inc.. Cynosure receives FDA clearance for PicoSure $^{\mathrm{TM}}$, the first picosecond device to remove tattoos and benign pigmented lesions [online]. [cited 2020 Jun 23]; Available from: URL: https://www.prnewswire.com/newsreleases/cynosure-receives-fda-clearance-for-picosure-thefirst-picosecond-device-to-remove-tattoos-and-benignpigmented-lesions-181993511.html

2. BOESH Group. Medical machines [online]. [cited 2020 Jun 23]; Available from: URL: http://www.boesh.de/pdfs/Catalog.pdf

3. Choi MS, Seo HS, Kim JG, Choe SJ, Park BC, Kim MH, et al. Effects of picosecond laser on the multi-colored tattoo removal using Hartley guinea pig: a preliminary study. PLoS One 2018;13:e0203370.

4. Bernstein EF, Schomacker KT, Shang X, Alessa D, Algzlan $\mathrm{H}$, Paranjape A. The first commercial $730 \mathrm{~nm}$ picoseconddomain laser is safe and effective for treating multicolor tattoos. Lasers Surg Med. Forthcoming 2020. https://doi. org/10.1002/Ism.23237

5. Saki N. Picosecond laser applications in aesthetic dermatology. J Surg Dermatol 2017;2(T1):107-8.

6. Kasai K. Picosecond laser treatment for tattoos and benign cutaneous pigmented lesions (secondary publication). Laser Ther 2017;26:274-81.

7. Supradeepa VR. Stimulated Brillouin scattering thresholds in 
optical fibers for lasers linewidth broadened with noise. Opt Express 2013;21:4677-87.

8. Dong J, Ueda K, Yagi H, Kaminskii AA. Laser-diode pumped self-Q-switched microchip lasers. Opt Rev 2008;15:57-74.

9. Tarasov A, Chu H. Generation of pulses with sub-nanosecond duration and sub-joule energy in gain-switched Ti: Sapphire lasers. Opt Express 2019;27:3574-82.

10. Kobyakov A, Sauer M, Chowdhury D. Stimulated Brillouin scattering in optical fibers. Adv Opt Photonics 2010;2:1-59.

11. Mungan CE, Rogers SD, Satyan N, White JO. Time-dependent modeling of brillouin scattering in optical fibers excited by a chirped diode laser. IEEE J Quantum Electron 2012;48:15426.

12. Jolly A, Gokhan FS, Bello R, Dupriez P. SBS management in $\mathrm{Yb}$-fiber-amplifiers using multimode seeds and pulse-shaping. Opt Express 2014;22:20326-46.

13. Dong L, Samson B. Fiber lasers: basics, technology, and applications. Boca Raton: CRC Press; 2017.

14. Teppitaksak A, Thomas GM, Damzen MJ. Investigation of a versatile pulsed laser source based on a diode seed and ultra-high gain bounce geometry amplifiers. Opt Express 2015;23:12328-36.

15. Liu X, He H, Song Y, Wang C, Wang Z. 500-kHz level high energy double-pass Nd:YVO4 picosecond amplifier with opticoptic efficiency of 51\%. Appl Sci 2019;9:219.

16. Zhang CH, Yao BQ, Li G, Wang Q, Ju YL, Wang YZ. 2041.3 $\mathrm{nm} / 2054.6 \mathrm{~nm}$ simultaneous dual-wavelength singlelongitudinal-mode Tm, Ho:YVO4 microchip laser. Laser Phys 2010;20:1564-7.

17. Henderson SW, Suni PJM, Hale CP, Hannon SM, Magee JR, Bruns DL, et al. Coherent laser radar at 2 mu $\mathrm{m}$ using solidstate lasers. IEEE Trans Geosci Remote Sens 1993;31:4-15.

18. Wang C, Shen L, Zhao Z, Liu B, Jiang $H$, Chen J, et al. 1.2 MW peak power, all-solid-state picosecond laser with a microchip laser seed and a high gain single-passing bounce geometry amplifier. Opt Laser Technol 2016;85:14-8.

19. SlidePlayer. Fundamentals of photonics 1 chapter 3 laser amplifiers [online]. [cited 2020 Jun 23]; Available from: URL: https://slideplayer.com/slide/5261043/

20. Hilborn RC. Einstein coefficients, cross sections, f values, dipole moments, and all that. Am J Phys 1982;50:982.

21. SlidePlayer. Laser amplifiers [online]. [cited 2020 Jun 23]; Available from: URL: https://slideplayer.com/slide/13872072/

22. Forget $S$, Balembois F, Georges P, Devilder PJ. A new 3D multipass amplifier based on Nd:YAG or Nd:YVO4 crystals. Appl Phys B 2002;75:481-5.

23. Tzuk $Y$, Glick $Y$, Tilleman MM, Kaufman A. Compact ultrahigh-gain multipass Nd:YAG amplifier with a low passive reflection phase-conjugate mirror. Proc SPIE 3263, Nonlinear Optical Engineering. Forthcoming 1998. https://doi. org/10.1117/12.308345 